\title{
A Note on Weighted Estimates for the Schrödinger Operator
}

\author{
Juan A. BARCEló, Jonathan M. BenNetT, \\ Anthony CARBERY, Alberto RUIZ, \\ and M. Cruz VILELA
}

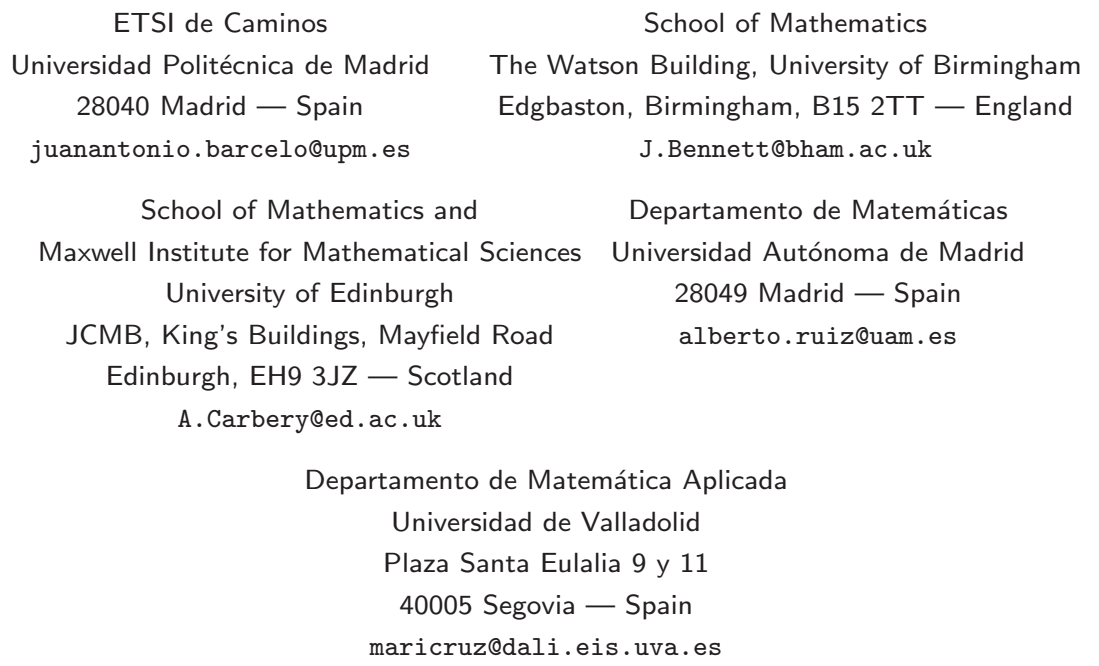

Received: October 3, 2007

Accepted: December 20, 2007

\begin{abstract}
We study two problems closely related to each other. The first one is concerned with some smoothing weighted estimates with weights in a certain MorreyCampanato spaces, for the solution of the free Schrödinger equation. The second one is a weighed trace inequality.

Key words: restriction theorem, Schrödinger operator.

2000 Mathematics Subject Classification: 42B15, 35J05.

The first and fourth were supported by Spanish Grant BFM2002-02204, the second by EPSRC Postdoctoral Fellowship GR/S27009/02, the third by a Leverhulme Study Abroad Fellowship and EC project "Pythagoras II" and the fifth by Spanish Grant MTM2004-03029.
\end{abstract}




\section{Introduction}

Consider the initial value problem associated to the free Schrödinger equation:

$$
\left\{\begin{array}{l}
i \partial_{t} u+\Delta_{x} u=0, \quad(x, t) \in \mathbb{R}^{n} \times \mathbb{R}, \\
u(x, 0)=f(x) .
\end{array}\right.
$$

A straightforward application of the Fourier transform allows us express the solution $u$ of (1) as

$$
u(x, t)=\int_{\mathbb{R}^{n}} e^{-\pi i t|\xi|^{2}+2 \pi i x \cdot \xi} \hat{f}(\xi) d \xi,
$$

where $\hat{f}$ is the Fourier transform of $f$. As usual, we denote this solution by $e^{i t \Delta} f(x)$.

In this work, we discuss a priori estimates for solutions of (1) that are related to weighted estimates which were used in [7] to treat the perturbation of the free equation by Morrey-Campanato time depending potentials $V$. This problem is closely related to a certain weighted restriction theorem for the Fourier transform of the measure on the unit sphere. This allow us to give partial answers to some open questions in $[1,5,7]$.

The Morrey-Campanato classes, see [8], which are denoted by $\mathcal{L}^{\alpha, p}$, for $\alpha>0$ and $1 \leq p \leq n / \alpha$, are given by

$$
\mathcal{L}^{\alpha, p}=\left\{V \in L_{\mathrm{loc}}^{p}\left(\mathbb{R}^{n}\right):\|V\|_{\mathcal{L}^{\alpha, p}}<\infty\right\},
$$

where

$$
\|V\|_{\mathcal{L}^{\alpha, p}}=\sup _{x \in \mathbb{R}^{n}, r>0} r^{\alpha}\left(r^{-n} \int_{B(x, r)}|V(y)|^{p} d y\right)^{1 / p} .
$$

Notice that $\mathcal{L}^{\alpha, n / \alpha}=L^{n / \alpha}\left(\mathbb{R}^{n}\right)$. We also remark that for $p<n / \alpha$ the class $\mathcal{L}^{\alpha, p}$ contains the Lorentz space $L^{n / \alpha, \infty}\left(\mathbb{R}^{n}\right)$.

Ruiz and Vega (see [7, Proposition 2.3]) proved the following estimates for the solution of the initial value problem (1):

$$
\left\|e^{i t \Delta} f\right\|_{L_{x, t}^{2}(V(x, t))} \leq C\left\|\sup _{t \in \mathbb{R}} V(x, t)\right\|_{\mathcal{L}_{x}^{2, p}}^{1 / 2}\|f\|_{L^{2}\left(\mathbb{R}^{n}\right)}
$$

for $(n-1) / 2<p \leq n / 2, p \geq 1$ and $n \geq 2$.

In the same paper they used the estimates (2) to solve the Cauchy problem associated to the Schrödinger equation with a potential $V(x, t) \in \mathcal{L}_{x}^{2, p} L_{t}^{\infty}$. Following their method, we extend the family of estimates (2) to the situation where the space $\mathcal{L}^{2, p}$ is replaced by $\mathcal{L}^{\alpha, p}$. The homogeneity of the Schrödinger equation forces us to introduce some $x$-derivatives of fractional order. We denote these as usual by $D_{x}^{\beta}$, and they are defined in the usual way via the Fourier transform. (Here, $\beta$ may be positive or negative, and in the latter case $D_{x}^{\beta}$ should be interpreted as fractional integration.) 
Theorem 1.1. Let $n \geq 2$ and let $V$ be a non-negative function in the space $\mathcal{L}_{x}^{\alpha, p} L_{t}^{\infty}$ with $\frac{2 n}{n+1}<\alpha \leq n, p \geq 1$ and $\frac{\alpha}{n} \leq \frac{1}{p}<\frac{2(\alpha-1)}{n-1}$. Then

$$
\left\|D_{x}^{1-\frac{\alpha}{2}} e^{i t \Delta} f\right\|_{L_{x, t}^{2}(V(x, t))} \leq C\left\|\sup _{t \in \mathbb{R}} V(x, t)\right\|_{\mathcal{L}_{x}^{\alpha, p}}^{1 / 2}\|f\|_{L^{2}\left(\mathbb{R}^{n}\right)} .
$$

Remark 1.2. Thus when $\alpha<2$ we have a smoothing effect with a gain of $1-\frac{\alpha}{2}$ $x$-derivatives.

Ruiz and Vega obtained the estimates (2) using the particular case $\alpha=2$ of the following restriction theorem (see [5, Theorem 2], and see also [3, 4]):

Theorem 1.3. Let be $n \geq 2, \mathbb{S}^{n-1}$ the unit sphere in $\mathbb{R}^{n}$, do the induced Lebesgue measure on $\mathbb{S}^{n-1}$ and $W$ a non-negative function defined on $\mathbb{R}^{n}$. If $\frac{2 n}{n+1}<\alpha \leq n$, $p \geq 1$, and $\frac{\alpha}{n} \leq \frac{1}{p}<\frac{2(\alpha-1)}{n-1}$, then there exists a positive constant $C=C(n, \alpha, p)$ such that

$$
\|\widehat{f d \sigma}\|_{L^{2}(W)} \leq C\|W\|_{\mathcal{L}^{\alpha, p}}^{1 / 2}\|f\|_{L^{2}\left(\mathbb{S}^{n-1}\right)} .
$$

We show that estimate (4) continues to imply estimate (3) when $\alpha \neq 0$. we are also interested in the necessary conditions in terms of the parameters $\alpha$ and $p$ for (3) and (4) to hold.

We prove that, in the case when there is a smoothing effect, Theorems 1.1 and 1.3 are sharp except for the border line $\frac{1}{p}=\frac{2(\alpha-1)}{n-1}$.

Theorem 1.4. Let $n \geq 2, \alpha<2$ and suppose that (3) or (4) holds. Then

$$
\frac{1}{p} \leq \frac{2(\alpha-1)}{n-1}
$$

We also have

Theorem 1.5. Let $n \geq 4, \alpha \geq 2$, and suppose that (3) or (4) hold. Then

$$
\frac{1}{p} \leq \frac{2 \alpha}{n}
$$

Remark 1.6. Theorem 1.5 contains a result which is similar to the one given in Proposition 3.4 of [1], but for the unit sphere in $\mathbb{R}^{n}$ instead of the section of the paraboloid

$$
\left\{\xi=\left(\xi^{\prime}, \xi_{n}\right) \in \mathbb{R}^{n-1} \times \mathbb{R}: \xi_{n}=\left|\xi^{\prime}\right|^{2} / 2,0 \leq \xi_{1}, \ldots, \xi_{n-1} \leq 1\right\} .
$$

In [1] we pointed out that the proof of the result for the mentioned section of the paraboloid do not appear to extend, in a routine manner, to other curved submanifolds of the type we considered there, as for example the unit sphere. As we will see in the proof of Theorem 1.1, any estimate of type (4) implies an estimate (3) for the same values of $\alpha$ and $p$. This allow us to get necessary conditions for the restriction of the Fourier transform (4) to hold by constructing counterexamples for the evolution Schrödinger equation (3), although the result that we get is not so good as the result obtained directly in [1] for the section of the paraboloid given in (5). 


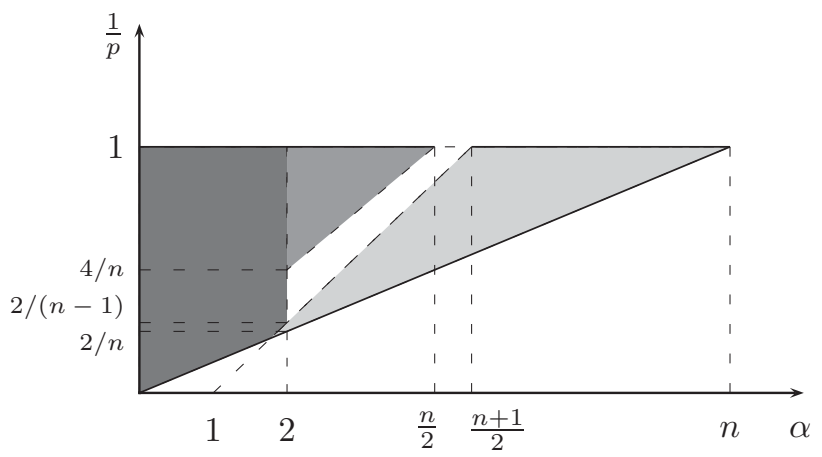

Figure 1 - For $n \geq 4$, the region with the lightest shade of grey corresponds to where the estimates (4) and (3) are known to be true, and the remaining darker shades correspond to where it is known to be false.

Figure 1 summarizes all the results that we present here.

Remark 1.7. The non-convexity of the regions $(\alpha, 1 / p)$ in above estimates may be possible due to the bad interpolation properties of Morrey-Campanato classes, see $[2,6]$.

\section{NotATION}

For non-negative quantities $X$ and $Y$ we use $X \lesssim Y(X \gtrsim Y)$ to denote the existence of a positive constant $C$, depending on at most $n$, such that $X \leq C Y(X \geq C Y)$. We write $X \sim Y$ if both $X \lesssim Y$ and $X \gtrsim Y$.

\section{Proofs}

Proof of Theorem 1.1. Following [7], we use polar coordinates and a change of variables to write

$$
D_{x}^{1-\frac{\alpha}{2}} e^{i t \Delta} f(x)=\widehat{g_{x}}(t)
$$

with

$$
g_{x}(r)=\frac{\chi_{(0, \infty)}(r)}{2 r^{\frac{\alpha}{4}}}\left(\hat{f} d \sigma_{\sqrt{r}} \hat{)}(x) .\right.
$$

Writing $W(x)=\sup _{t \in \mathbb{R}} V(x, t)$, and using Plancherel's identity we obtain

$$
\begin{aligned}
\left\|D_{x}^{1-\frac{\alpha}{2}} e^{i t \Delta} f\right\|_{L_{x, t}^{2}(V(x, t))} & \lesssim\|\| g_{x}(r)\left\|_{L_{r}^{2}}\right\|_{L_{x}^{2}(W)} \\
& \sim\left(\int_{0}^{r} r^{-\frac{\alpha}{2}} \|\left(\hat{f} d \sigma_{\sqrt{r}} \hat{(}(x) \|_{L_{x}^{2}(W)}^{2} d r\right)^{1 / 2} .\right.
\end{aligned}
$$


On the other hand, from (4) we have that

$$
\left\|\widehat{f d \sigma_{\sqrt{r}}}\right\|_{L^{2}(W)} \lesssim r^{\frac{(\alpha-1)}{4}}\|W\|_{\mathcal{L}^{\alpha, p}}^{1 / 2}\|f\|_{L^{2}\left(\mathbb{S}_{\sqrt{r}}^{n-1}\right)} .
$$

Using this estimate in (6), making a change of variables, using polar coordinates and Plancherel's identity, we obtain

$$
\begin{aligned}
\left\|D_{x}^{1-\frac{\alpha}{2}} e^{i t \Delta} f\right\|_{L_{x, t}^{2}(V(x, t))} & \lesssim\|W\|_{\mathcal{L}^{\alpha, p}}^{1 / 2}\left(\int_{0}^{r} r^{-1 / 2}\|\hat{f}\|_{L^{2}\left(S_{\sqrt{r}}^{n-1}\right)}^{2} d r\right)^{1 / 2} \\
& \sim\left\|\sup _{t \in \mathbb{R}} V(x, t)\right\|_{\mathcal{L}_{x}^{\alpha, p}}^{1 / 2}\|f\|_{L^{2}\left(\mathbb{R}^{n}\right)}
\end{aligned}
$$

Proof of Theorem 1.4. This result for the estimate (4) was proved in [1, Lemma 3.3] by using standard counterexamples, that is, the characteristic funcion of a $\delta$-cell on $\mathbb{S}^{n-1}$. But these standard counterexamples do not work for the estimates (3) because of the homogeneity. As substitutes, we consider the same kind of counterexamples that work for the Strichartz estimates (see [9]).

Take $\hat{f}(\xi)=\sum_{j=0}^{M} \hat{f}_{j}(\xi)$ where $M$ is a positive number as large as we need and

$$
\hat{f}_{j}(\xi)=e^{i j^{2} M^{2}|\xi|^{2}} \varphi\left(\xi_{1}-j M\right) \prod_{\ell=2}^{n} \varphi\left(\xi_{\ell}\right)
$$

with $\varphi \in C_{0}^{\infty}(\mathbb{R})$ such that $\operatorname{supp} \varphi=[-1,1]$ and $0 \leq \varphi \leq 1$. For each $f_{j}$ we can write

$$
\begin{aligned}
\left|D_{x}^{1-\frac{\alpha}{2}} e^{i t \Delta} f_{j}(x)\right| & =\int_{\mathbb{R}^{n}}|\xi|^{1-\frac{\alpha}{2}} e^{-\pi i t|\xi|^{2}+2 \pi i x \cdot \xi} \widehat{f}_{j}(\xi) d \xi \\
& \sim(j M)^{1-\frac{\alpha}{2}}\left|\int_{\mathbb{R}} \varphi\left(\xi_{1}-j M\right) e^{i \phi_{1}^{j}\left(\xi_{1}\right)} d \xi_{1} \prod_{\ell=2}^{n} \int_{\mathbb{R}} \varphi\left(\xi_{\ell}\right) e^{i \phi_{\ell}^{j}\left(\xi_{\ell}\right)} d \xi_{\ell}\right|,
\end{aligned}
$$

where $\phi_{\ell}^{j}\left(\xi_{\ell}\right)=-\pi\left(t-j^{2} M^{2}\right) \xi_{\ell}^{2}+2 \pi x_{\ell} \xi_{\ell}$ for $\ell=1, \ldots, n$. Making a change of variable, we have that

$$
\left|D_{x}^{1-\frac{\alpha}{2}} e^{i t \Delta} f_{j}(x)\right| \sim(j M)^{1-\frac{\alpha}{2}}\left|\int_{\mathbb{R}} \varphi\left(\xi_{1}\right) e^{i \psi^{j}\left(\xi_{1}\right)} d \xi_{1} \prod_{\ell=2}^{n} \int_{\mathbb{R}} \varphi\left(\xi_{\ell}\right) e^{i \phi_{\ell}^{j}\left(\xi_{\ell}\right)} d \xi_{\ell}\right|,
$$

with $\psi^{j}\left(\xi_{1}\right)=-\pi\left(t-j^{2} M^{2}\right) \xi_{1}^{2}+2 \pi\left[x_{1}-j M\left(t-j^{2} M^{2}\right)\right] \xi_{1}$.

For $j=0, \ldots, M$, we define

$$
\begin{aligned}
B_{j}=\left\{\left(x_{1}, \ldots, x_{n}, t\right)\right. & \in \mathbb{R}^{n+1} \text { such that } \\
\left|t-j^{2} M^{2}\right| & \left.\leq 1,\left|x_{1}-j M\left(t-j^{2} M^{2}\right)\right| \leq 1, \quad\left|x_{\ell}\right| \leq 1, \quad \ell=2, \ldots, n\right\} .
\end{aligned}
$$


Under these conditions, if $(x, t) \in B_{j}$, then $\left|\psi^{j}\left(\xi_{1}\right)\right| \lesssim 1$, for all $\xi_{1} \in \operatorname{supp} \varphi$, and $\left|\phi_{\ell}^{j}\left(\xi_{\ell}\right)\right| \lesssim 1$, for all $\xi_{\ell} \in \operatorname{supp} \varphi, \ell=2, \ldots, n$. Hence

$$
\left|D_{x}^{1-\frac{\alpha}{2}} e^{i t \Delta} f_{j}(x)\right| \sim(j M)^{1-\frac{\alpha}{2}} \quad \text { if } \quad(x, t) \in B_{j} .
$$

On the other hand, for $k \neq j$, if $(x, t) \in B_{k}$, then $\left|\left(\psi^{j}\right)^{\prime}\left(\xi_{1}\right)\right| \gtrsim M$, for all $\xi_{1} \in$ $\operatorname{supp} \varphi$. Thus, integrating by parts we obtain that, for any $m \in \mathbb{N}$,

$$
\left|D_{x}^{1-\frac{\alpha}{2}} e^{i t \Delta} f_{j}(x)\right| \sim M^{-m} \quad \text { if } \quad(x, t) \in B_{k} \quad \text { with } \quad k \neq j .
$$

Therefore, if we take $V$ as the characteristic function of the set $B=\cup_{j=0}^{M} B_{j}$, since $\alpha \leq 2$, we have that

$$
\begin{aligned}
\left\|D_{x}^{1-\frac{\alpha}{2}} e^{i t \Delta} f\right\|_{L_{x, t}^{2}(V)} & =\left(\sum_{j=0}^{M} \int_{B_{j}}\left|\sum_{k=0}^{M} D^{1-\frac{\alpha}{2}} e^{i t \Delta} f_{k}(x)\right|^{2} d x d t\right)^{1 / 2} \\
& \sim M^{1-\frac{\alpha}{2}}\left(\sum_{j=0}^{M} j^{2-\alpha}\right)^{1 / 2} \sim M^{\frac{5}{2}-\alpha} .
\end{aligned}
$$

On the other hand, writing $A_{j}=\operatorname{supp} \hat{f}_{j}$ for $j=0, \ldots, M$,

$$
\|f\|_{L^{2}\left(\mathbb{R}^{n}\right)}=\left(\sum_{j=0}^{M} \int_{A_{j}}\left|\sum_{k=0}^{M} \hat{f}_{k}(\xi)\right|^{2} d \xi\right)^{1 / 2} \sim M^{1 / 2} .
$$

Finally, $\sup _{t \in \mathbb{R}} V(x, t)=\chi_{C}(x)$ with

$$
C=\left\{\left(x_{1}, \ldots, x_{n}\right) \in \mathbb{R}^{n}:\left|x_{1}\right| \lesssim M^{2},\left|x_{\ell}\right| \lesssim 1, \ell=2, \ldots, n\right\},
$$

and thus

$$
\left\|\sup _{t \in \mathbb{R}} V(x, t)\right\|_{\mathcal{L}_{x}^{\alpha, p}} \sim \begin{cases}1, & \text { if } p \leq(n-1) / \alpha, \\ M^{2 \alpha-2(n-1) / p}, & \text { if } p \geq(n-1) / \alpha .\end{cases}
$$

The result follows from (7), (8), and (9) taking $M$ large enough.

The previous counterexamples give negative results just for $\alpha<2$, now we will use some special solutions of the problem (1), introduced in [1], to get negative results for $\alpha \geq 2$.

Proof of Theorem 1.5. As we mentioned in Remark 1.6, (4) implies (3). Therefore, it is enough to prove the result for the estimate (3).

Following [1], let $0<\delta \ll 1$ and $0<\sigma<\frac{1}{2}$. We consider the function of one variable

$$
g=\sum_{\substack{\ell \in \mathbb{N} \\ 1 \leq \ell \leq \delta^{-\sigma}}} \chi_{\left(\ell \delta^{\sigma}-\delta, \ell \delta^{\sigma}+\delta\right)} .
$$


Note that $g$ is simply the characteristic function of a union of disjoint, equally spaced subintervals of $[0,1]$ of equal size. We now define $f$ by

$$
\hat{f}(\xi)=\prod_{j=1}^{n} g\left(\xi_{j}\right),
$$

where $\xi=\left(\xi_{1}, \ldots, \xi_{n}\right)$. of

We now set $X=\left\{p \delta^{-\sigma}: p \in \mathbb{N}\right.$ with $\left.p \lesssim \delta^{\sigma-1}\right\}$, and $\Omega$ to be an $O(1)$-neighborhood

$$
\Lambda=\left\{(x, t) \in \mathbb{R}^{n} \times \mathbb{R}: x \in X^{n} \text { and } t=2 q \delta^{-2 \sigma} \text { where } q \in \mathbb{N} \text { and } q \lesssim \delta^{2 \sigma-1}\right\},
$$

and $V$ as the characteristic function of the set $\Omega$.

Arguing as in [1], for $(x, t) \in \Omega$ we have the uniform bound

$$
\begin{aligned}
\left|D_{x}^{1-\frac{\alpha}{2}} e^{i t \Delta} f(x)\right| & =\left.\left|\int_{\mathbb{R}^{n}}\right| \xi\right|^{1-\frac{\alpha}{2}} e^{-\pi i t|\xi|^{2}+2 \pi i x \cdot \xi} \hat{f}(\xi) d \xi \mid \\
& \gtrsim \int_{\left[\frac{1}{2}, 1\right]^{n}} \hat{f}(\xi) d \xi \sim \delta^{n(1-\sigma)},
\end{aligned}
$$

and hence,

$$
\left\|D_{x}^{1-\frac{\alpha}{2}} e^{i t \Delta} f\right\|_{L_{x, t}^{2}(V)} \gtrsim \delta^{n(1-\sigma)}|\Omega|^{\frac{1}{2}}=\delta^{(1-\sigma) \frac{n}{2}+\sigma-\frac{1}{2}} .
$$

On the other hand,

$$
\|f\|_{L^{2}\left(\mathbb{R}^{n}\right)}=\|\hat{f}\|_{L^{2}\left(\mathbb{R}^{n}\right)} \sim \delta^{(1-\sigma) \frac{n}{2}}
$$

and for $1 \leq p \leq n / \alpha$,

$$
\left\|\sup _{t \in \mathbb{R}} V(x, t)\right\|_{\mathcal{L}_{x}^{\alpha, p}} \sim \max \left\{1, \delta^{\frac{\sigma n}{p}-\alpha}\right\}
$$

Taking into account that $\delta$ is a very small positive number and $0<\sigma<1 / 2$, we obtain the result from (10), (11), and (12).

\section{References}

[1] J. A. Barceló, J. M. Bennett, A. Carbery, A. Ruiz, and M. C. Vilela, Some special solutions of the Schrödinger equation, Indiana Univ. Math. J. 56 (2007), no. 4, 1581-1593.

[2] O. Blasco, A. Ruiz, and L. Vega, Non-interpolation in Morrey-Campanato and block spaces, Ann. Scuola Norm. Sup. Pisa Cl. Sci. (4) 28 (1999), no. 1, 31-40.

[3] S. Chanillo and E. Sawyer, Unique continuation for $\Delta+V$ and the C. Fefferman-Phong class, Trans. Amer. Math. Soc. 318 (1990), no. 1, 275-300.

[4] F. Chiarenza and A. Ruiz, Uniform $L^{2}$-weighted Sobolev inequalities, Proc. Amer. Math. Soc. 112 (1991), no. 1, 53-64. 
[5] A. Ruiz and L. Vega, Unique continuation for Schrödinger operators with potential in Morrey spaces, Publ. Mat. 35 (1991), no. 1, 291-298. Conference on Mathematical Analysis (El Escorial, 1989).

[6] _ Corrigenda to: "Unique continuation for Schrödinger operators with potential in Morrey spaces" and a remark on interpolation of Morrey spaces, Publ. Mat. 39 (1995), no. 2, 405-411.

[7] _ Local regularity of solutions to wave equations with time-dependent potentials, Duke Math. J. 76 (1994), no. 3, 913-940.

[8] G. Stampacchia, $\mathcal{L}^{(p, \lambda)}$-spaces and interpolation, Comm. Pure Appl. Math. 17 (1964), 293-306.

[9] M. C. Vilela, Inhomogeneous Strichartz estimates for the Schrödinger equation, Trans. Amer. Math. Soc. 359 (2007), no. 5, 2123-2136. 